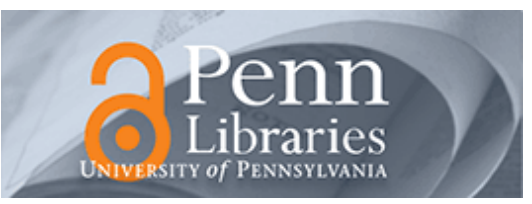

University of Pennsylvania

ScholarlyCommons

6-1-2010

\title{
Single-Ended-to-Differential and Differential-to-Differential Channel-Select Filters Based on Piezoelectric AIN Contour-Mode MEMS Resonators
}

\author{
Chengjie Zuo \\ University of Pennsylvania, chengjiezuo@hotmail.com \\ Gianluca Piazza \\ University of Pennsylvania, piazza@seas.upenn.edu
}

Follow this and additional works at: https://repository.upenn.edu/ese_papers

Part of the Electrical and Electronics Commons

\section{Recommended Citation}

Chengjie Zuo and Gianluca Piazza, "Single-Ended-to-Differential and Differential-to-Differential ChannelSelect Filters Based on Piezoelectric AIN Contour-Mode MEMS Resonators", 2010 IEEE International Frequency Control Symposium (IFCS 2010) , 5-8. June 2010.

Suggested Citation:

Zuo, Chengji and Gianluca Piazza. "Single-Ended-to-Differential and Differential-to-Differential Channel-Select Filters Based on Piezoelectric AIN Contour-Mode MEMS Resonators for Multi-Frequency Reconfigurable RF Transceivers" IEEE Solid-State Circuits Magazine Vol. 2 p. 74-75.

"C2010 IEEE. Personal use of this material is permitted. However, permission to reprint/republish this material for advertising or promotional purposes or for creating new collective works for resale or redistribution to servers or lists, or to reuse any copyrighted component of this work in other works must be obtained from the IEEE."

This paper is posted at ScholarlyCommons. https://repository.upenn.edu/ese_papers/548

For more information, please contact repository@pobox.upenn.edu. 


\title{
Single-Ended-to-Differential and Differential-to-Differential Channel-Select Filters Based on Piezoelectric AIN Contour-Mode MEMS Resonators
}

\author{
Abstract \\ This paper reports on the first demonstration of single-ended-to-differential and differential-to-differential \\ (S2D and D2D) channel-select filters based on single-layer (SL) and dual-layer-stacked (DLS) AIN contour- \\ mode MEMS resonators. The key filter performances in terms of insertion loss (as low as $1.4 \mathrm{~dB}$ ), \\ operating frequency (250-1280 MHz), and out-of-band rejection (up to $60 \mathrm{~dB}$ ) constitute a significant \\ advancement over all other state-of-the-art RF MEMS technologies. The fabrication process, namely \\ stacking of two piezoelectric AIN layers (600 nm each) and three Pt electrode layers (100 nm each), is \\ fully compatible with the previously demonstrated AIN RF MEMS switch process (also post-CMOS \\ compatible), which makes it possible to implement multi-frequency switchable filter banks on a single \\ chip. The S2D configuration is also able to combine the balun, filter, and impedance transformer functions \\ in a single MEMS structure and only takes on a very small form factor $(60 \times 200 \mu \mathrm{m})$. These unique \\ features will potentially revolutionize the field of RF and microwave IC design by enabling MEMS-IC co- \\ design and the development of unconventional and low-power RF architectures.

\section{Disciplines} \\ Electrical and Electronics

\section{Comments} \\ Suggested Citation: \\ Zuo, Chengji and Gianluca Piazza. "Single-Ended-to-Differential and Differential-to-Differential Channel- \\ Select Filters Based on Piezoelectric AIN Contour-Mode MEMS Resonators for Multi-Frequency \\ Reconfigurable RF Transceivers" IEEE Solid-State Circuits Magazine Vol. 2 p. 74-75. \\ "(C2010 IEEE. Personal use of this material is permitted. However, permission to reprint/republish this \\ material for advertising or promotional purposes or for creating new collective works for resale or \\ redistribution to servers or lists, or to reuse any copyrighted component of this work in other works must \\ be obtained from the IEEE."
}




\title{
Single-Ended-to-Differential and Differential-to- Differential Channel-Select Filters Based on Piezoelectric AlN Contour-Mode MEMS Resonators
}

\author{
Chengjie Zuo and Gianluca Piazza \\ Department of Electrical and Systems Engineering \\ University of Pennsylvania \\ Philadelphia, PA 19104, USA
}

\begin{abstract}
This paper reports on the first demonstration of single-ended-to-differential and differential-to-differential (S2D and D2D) channel-select filters based on single-layer (SL) and dual-layer-stacked (DLS) AIN contour-mode MEMS resonators. The key filter performances in terms of insertion loss (as low as $1.4 \mathrm{~dB})$, operating frequency $(250-1280 \mathrm{MHz})$, and out-of-band rejection (up to $60 \mathrm{~dB}$ ) constitute a significant advancement over all other state-of-the-art RF MEMS technologies. The fabrication process, namely stacking of two piezoelectric AIN layers $(600 \mathrm{~nm}$ each) and three Pt electrode layers $(100 \mathrm{~nm}$ each $)$, is fully compatible with the previously demonstrated AIN RF MEMS switch process (also post-CMOS compatible), which makes it possible to implement multi-frequency switchable filter banks on a single chip. The S2D configuration is also able to combine the balun, filter, and impedance transformer functions in a single MEMS structure and only takes on a very small form factor $(60 \times 200 \mu \mathrm{m})$. These unique features will potentially revolutionize the field of RF and microwave IC design by enabling MEMS-IC co-design and the development of unconventional and low-power RF architectures.
\end{abstract}

Index Terms - Channel-select filters, microelectromechanical systems (MEMS), AIN contour-mode resonators, piezoelectric resonators, dual-layer-stacked AIN, single-ended-to-differential filters, differential-to-differential filters.

\section{INTRODUCTION}

A promising solution for future single-chip multi-frequency reconfigurable wireless communications [1], and especially low-power wireless sensor network applications [2], is based on the use of arrays of switchable narrow-band filters closely spaced in frequency so as to realize channel selection directly at the radio frequency (RF) stage [3]. Simultaneously, most of the on-chip RF solutions rely on differential signal processing in order to reject common-mode noise and reduce sensitivity to supply voltage fluctuations.

Available mechanical filters such as surface acoustic wave (SAW) devices are capable of realizing differential solutions, but are bulky, non-integrable with silicon substrates (i.e., CMOS electronics), and can hardly span a broad frequency range on the same substrate due to the dependence of the electromechanical coupling on the metallization thickness (i.e., the need of multiple fabrication steps or packages). Film Bulk Acoustic Resonators (FBARs) [4] can only have one frequency per wafer (since the film thickness sets their resonant frequency) and are intrinsically single-ended devices (since they usually cannot have multiple electrodes patterned on the same surface). On the other hand, more recent demonstrations of single-ended-to-differential and differential-to-differential (S2D and D2D) filters with FBARs [5] require a complicated manufacturing process that takes advantage of additional mechanical coupling layers and limits the overall order of the filter.

Promising electrostatically-transduced silicon-based micro electromechanical (MEMS) [1] S2D and D2D solutions have also been proposed recently, but they either operate at low frequencies and require large termination impedances and complex mechanical links [6] or suffer from large insertion loss in excess of $8 \mathrm{~dB}$ [7].

In order to address these existing challenges, we report on the design and experimental demonstration of ultra-compact $(60 \times 200 \mu \mathrm{m})$, multi-frequency (from $250 \mathrm{MHz}$ to $1.28 \mathrm{GHz}$ ) and low-insertion-loss (as low as $1.4 \mathrm{~dB}$ for a $1^{\text {st }}$ order filter at $253 \mathrm{MHz}$ ) S2D and D2D channel-select filters based on postCMOS compatible, MEMS Aluminum Nitride (AIN) contourmode resonators (CMRs) [8-9] (Figs. 1 and 4). By engineering the electrode configuration in single-layer (SL) and dual-layerstacked (DLS) piezoelectric AlN films (Figs. 1 and 2), the AlN CMRs combine the simplicity of SAW technology in terms of synthesizing differential solutions, with the CMOS integrability of silicon devices and the low impedance of piezoelectric FBARs. In addition, the piezoelectric AIN CMRs are laterally vibrating MEMS structures whose resonant frequency can be set lithographically and can therefore span a broad range of frequencies on the same chip (e.g., $250 \mathrm{MHz}-1.28 \mathrm{GHz}$ in this work). Finally, the S2D electrode configuration combines the functions of a filter (narrowband channel selection), a balun (single-ended to differential transformation), and an impedance transformer in an ultra-compact MEMS structure.

\section{SINGLE-LAYER FILTERS}

With a single-layer (SL) piezoelectric AlN film sandwiched between two Pt layers, the S2D and D2D configurations can be implemented by patterning the top and bottom Pt electrodes in a way so that the adjacent sub-resonators vibrate out of phase with respect to each other and provide the required opposite signal polarities for electrical differential operation (Fig. 1). The SL S2D and D2D configurations can be viewed as two conventional single-ended 2-port AIN contour-mode resonators [10] placed in parallel side by side. In the S2D and D2D implementations of this work, the two 2-port resonators are also mechanically coupled at the common edge so that the 

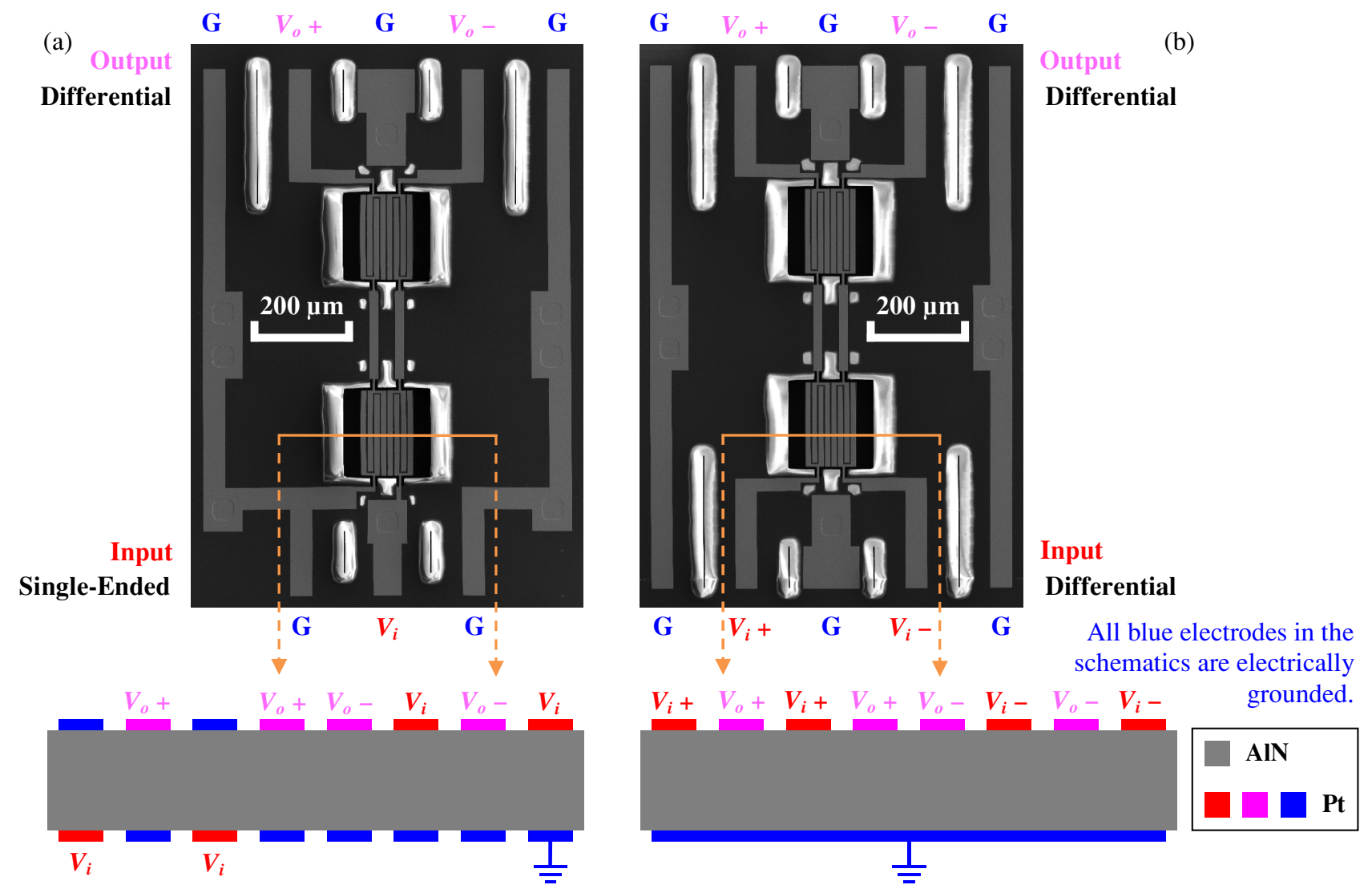

Fig. 1. SEM pictures and cross-sectional schematics for the $2^{\text {nd }}$ order (a) single-ended-to-differential (S2D) and (b) differential-to-differential (D2D) channel-select RF MEMS filters.

entire MEMS structure vibrates as a single body, therefore eliminating the problem associated with frequency mismatch that would exist with two separate resonators.

Using the same analysis as in [10], the AlN plate is treated as multiple AlN sub-resonators mechanically coupled together to form a single resonant body. In this way, a $1^{\text {st }}$ order S2D (D2D) filter can also be considered as a 3-port (4-port) AIN contour-mode MEMS resonator. The detailed analytical and equivalent circuit modeling of the 3-port and 4-port resonators follows the same principles described in [10], and therefore will not be repeated here.

\section{DUAL-LAYER-STACKED FILTERS}

In order to provide more freedom in electrode routing and simultaneously reduce the number of tethers (therefore anchor loss) connecting the resonator to the $\mathrm{Si}$ substrate, dual-layerstacked (DLS) S2D and D2D channel-select filters have also been fabricated, and tested. As shown in Fig. 2, DLS devices are formed by two layers of AIN thin film (600 $\mathrm{nm}$ each) and three $\mathrm{Pt}$ layers $(100 \mathrm{~nm}$ each for bottom, middle and top electrodes). The middle Pt layer is lithographically patterned and, if desired, can be omitted so as to provide the single-layer device option described in Section II.

The cross-sectional schematics for the corresponding DLS electrode configurations are given in Fig. 2. When compared with the SL counterparts, the DLS filter configuration achieves the same S2D and D2D operations within a half MEMS structure volume (and also chip area), and the number of required tethers to suspend the resonator is two instead of four. For optimum filter performance, piezoelectric filters usually need to be terminated by a proper resistance, which is approximately equal to the magnitude of the corresponding input (output) transducer impedance. Given the piezoelectric transducer thickness is halved and the number of fingers (subresonators) is doubled for each single-ended port in a DLS solution with respect to a SL, the DLS S2D and D2D filters require a lower termination resistance (approximately $1 / 4$ ) than the SL counterparts for the same chip area.

\section{EXPERIMENTAL RESULTS}

The filters were fabricated using a post-CMOS compatible process, which had also been used to integrate piezoelectric AlN RF MEMS switches capable of switching the CMRs on and off [11]. All the fabricated devices were directly probed in ambient conditions in a Desert Cryogenics ${ }^{\circledR}$ TTP6 probe station and measured by an Agilent ${ }^{\circledR}$ N5230A 4-port network analyzer after a complete 4-port calibration. The experimental results are listed in Table I, and they are net device performances without any de-embedding.

Table I shows that these S2D and D2D filters are capable of spanning a wide frequency range (from 250 to $1281 \mathrm{MHz}$ ) on the same silicon chip, with low insertion loss and good out of band rejection ( $>20 \mathrm{~dB}$ at most frequencies) even if solely 1 or 2 stages are used. In particular, the best performance ever reported for $2^{\text {nd }}$ order S2D and D2D channel-select MEMS 
filters has been achieved. Insertion loss as low as $1.8 \mathrm{~dB}$ (for a $2^{\text {nd }}$ order filter), narrow bandwidth around $0.5 \%$, high out-ofband rejection up to $60 \mathrm{~dB}$, and common-mode suppression (CMS) up to $35 \mathrm{~dB}$ have been attained. In addition, low insertion loss $\left(<2 \mathrm{~dB}\right.$, shown in Table I) $1^{\text {st }}$ order D2D filters have also been demonstrated up to $947 \mathrm{MHz}$, the highest frequency ever reported for differential MEMS filters based on laterally vibrating resonators. By electrically cascading these $1^{\text {st }}$ order filters and coupling them using the intrinsic capacitance of the piezoelectric AlN transducers [10], better rejection, common-mode suppression, and shape factor can be achieved as has been demonstrated in the $2^{\text {nd }}$ order $253 \mathrm{MHz}$ filter.

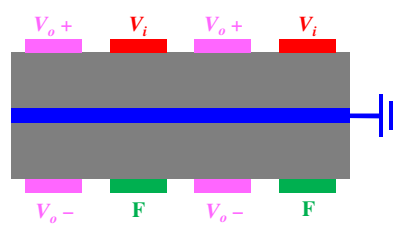

Single-ended to Differential

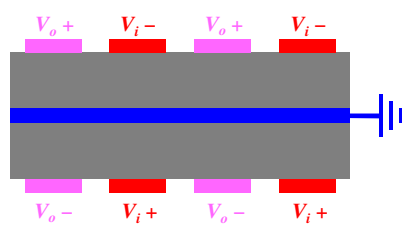

Differential to Differential

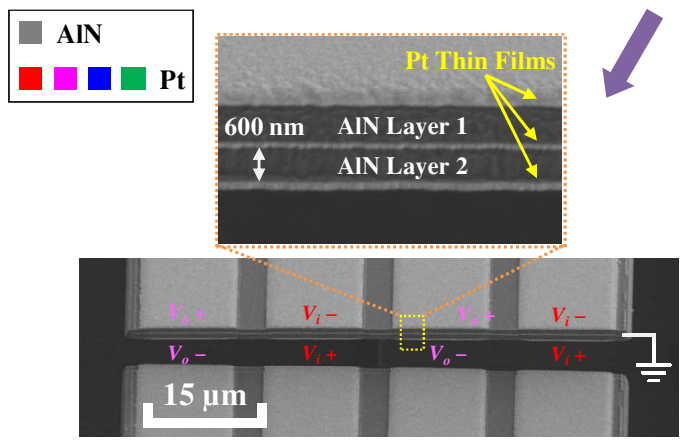

Fig. 2. Cross-sectional schematics and SEM pictures of dual-layerstacked (DLS) S2D and D2D channel-select filters. "F" stands for floating electrodes.

TABLE I. EXPERIMENTAL RESULTS OF S2D AND D2D FILTERS

\begin{tabular}{|c|c|c|c|c|c|c|c|c|}
\hline Filter & $\begin{array}{c}\boldsymbol{f}_{\boldsymbol{c}} \\
{[\mathbf{M H z}]}\end{array}$ & $\begin{array}{c}\boldsymbol{I L} \\
{[\mathbf{d B}]}\end{array}$ & $\boldsymbol{F B W _ { 3 \mathrm { dB } }}$ & $\begin{array}{c}\boldsymbol{R e j} \\
{[\mathbf{d B}]}\end{array}$ & $\begin{array}{c}\boldsymbol{C M S} \\
{[\mathbf{d B}]}\end{array}$ & Order & $\begin{array}{c}\boldsymbol{R}_{\boldsymbol{T}} \\
{[\boldsymbol{\Omega}]}\end{array}$ & $\boldsymbol{S F}_{\text {20dB }}$ \\
\hline SL + S2D & 272 & 1.5 & $0.47 \%$ & 23 & 15 & 1 & 2000 & 6.1 \\
\hline SL + S2D & 272 & 2.5 & $0.45 \%$ & 53 & 33 & 2 & 2000 & 3.1 \\
\hline SL + D2D & 253 & 1.4 & $0.59 \%$ & 27 & 23 & 1 & 2000 & 10.6 \\
\hline SL + D2D & 253 & 1.8 & $0.53 \%$ & 60 & 35 & 2 & 2000 & 3.2 \\
\hline SL + D2D & 947 & 1.9 & $0.26 \%$ & 21 & 24 & 1 & 1000 & 26.8 \\
\hline SL + D2D & 947 & 6.2 & $0.24 \%$ & 35 & 30 & 2 & 800 & 5.3 \\
\hline DLS + S2D & 250 & 1.9 & $0.41 \%$ & 28 & 14 & 1 & 1200 & 10.2 \\
\hline DLS + S2D & 681 & 5.3 & $0.19 \%$ & 30 & 18 & 1 & 700 & 17.6 \\
\hline DLS + S2D & 1281 & 6.6 & $0.37 \%$ & 17 & 10 & 1 & 600 & 25.5 \\
\hline DLS + D2D & 249 & 2.0 & $0.50 \%$ & 28 & 18 & 1 & 1200 & 8.9 \\
\hline DLS + D2D & 681 & 2.6 & $0.30 \%$ & 25 & 31 & 1 & 700 & 11.1 \\
\hline DLS + D2D & 996 & 4.1 & $0.44 \%$ & 27 & 16 & 1 & 500 & 14.7 \\
\hline
\end{tabular}

SL: single-layer AIN filters; DLS: dual-layer-stacked AIN filters; S2D: singleended-to-differential filters; D2D: differential-to-differential filters; $f_{c}$ : filter center frequency; $I L$ : insertion loss; $F B W_{3 \mathrm{~dB}}: 3 \mathrm{~dB}$ fractional bandwidth; Rej: out-of-band rejection; $C M S$ : common-mode suppression; Order: filter order; $R_{T}$ : termination resistance; $S F_{20 \mathrm{~dB}}: 20 \mathrm{~dB}$ shape factor; (Note: for the

"SL+S2D" filters, the single-ended port is terminated by a half $R_{T}$ )

Together with these SL filters and on the same chip, DLS $1^{\text {st }}$ order filters have also been demonstrated with frequencies of operation up to $1.28 \mathrm{GHz}$ and similar high performance. In these devices, the insertion loss deteriorates at high frequencies (still $<7 \mathrm{~dB}$ at $1.28 \mathrm{GHz}$ ) due to the additional (two AlN layer depositions) fabrication complexity required to synthesize DLS devices and the misalignment error that occurred during this specific fabrication run $(>500 \mathrm{~nm})$. As has been explained in Section III, when compared with the SL counterparts (Fig. 1) at the same frequency, the DLS filters advantageously use only $1 / 4$ of the chip area assuming a certain required termination resistance, which can be ultimately lowered to $50 \Omega$ for direct impedance matching with conventional RF electronics.
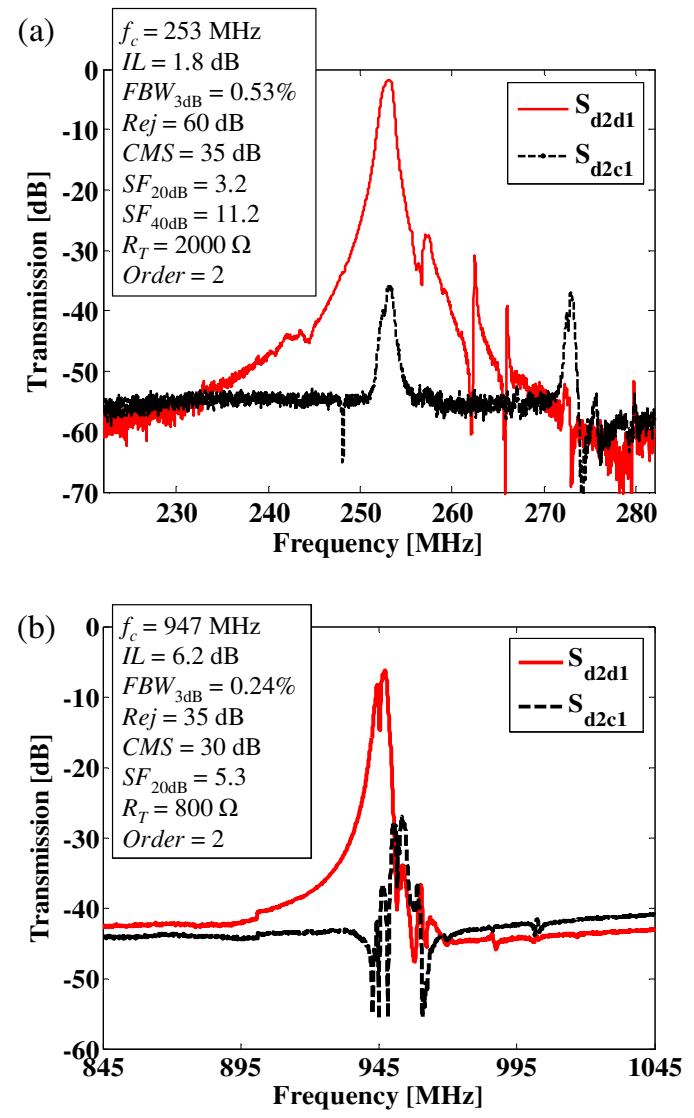

Fig. 3. Measured transmission responses for the $2^{\text {nd }}$ order SL D2D channel-select MEMS filters at (a) $253 \mathrm{MHz}$ and (b) $947 \mathrm{MHz}\left(\mathrm{S}_{\mathrm{d} 2 \mathrm{~d} 1}\right.$ : transmission from differential signal at port 1 to port $2 ; \mathrm{S}_{\mathrm{d} 2 \mathrm{cl}}$ : transmission from common-mode signal at port 1 to differential signal at port 2).

As two examples, the $S$-parameter data for the $2^{\text {nd }}$ order SL D2D channel-select MEMS filters at $253 \mathrm{MHz}$ and $947 \mathrm{MHz}$ are plotted in Fig. 3 (a) and (b), respectively. Due to the smaller electrode size (a few $\mu \mathrm{m})$, filters at higher frequencies are more vulnerable to misalignments and mismatches in the fabrication. In particular, this specific fabrication run suffered from large misalignment errors in excess of $500 \mathrm{~nm}$, which translated in relatively higher insertion loss and distorted passband in high frequency devices (Fig. 3 (b)). The smaller peaks existing in the signal transmission from common-mode to differential (basically non-ideal common-mode suppression) are believed to be caused by the observed $1 \mu \mathrm{m}$ misalignment (by SEM imaging) for the specific die under test. 


\section{APPLICATIONS}

The demonstrated multi-frequency operation, low insertion loss, and high out-of-band rejection make these MEMS filters very suitable for channel selection at the RF or IF stages of wireless communication systems, which can potentially address the well-known difficulty in tuning high- $Q$ systems by using switchable filter banks as described in $[1,3,12]$.

In addition, the SL S2D electrode configuration shown in Fig. 1 (a) was designed with the specific intent of synthesizing transformer, balun and filter, three different functionalities in a single component. The transducer capacitance at the singleended input port (denoted by $V_{i}$ ) is two times that of either the positive or the negative port (denoted by $V_{o}+$ and $V_{o}-$, respectively) at the differential output. Since the filter termination resistance is generally equal to the magnitude of transducer impedance, there exists an intrinsic impedance transformation between the single-ended input and the differential output. Therefore this SL S2D configuration combines the functions of a balun (single-ended to differential transformation), a filter (narrow-band channel selection) and an impedance transformer in an ultra-compact AlN structure. The same principle can also be implemented with the DLS AIN configuration by proper electrode routing.

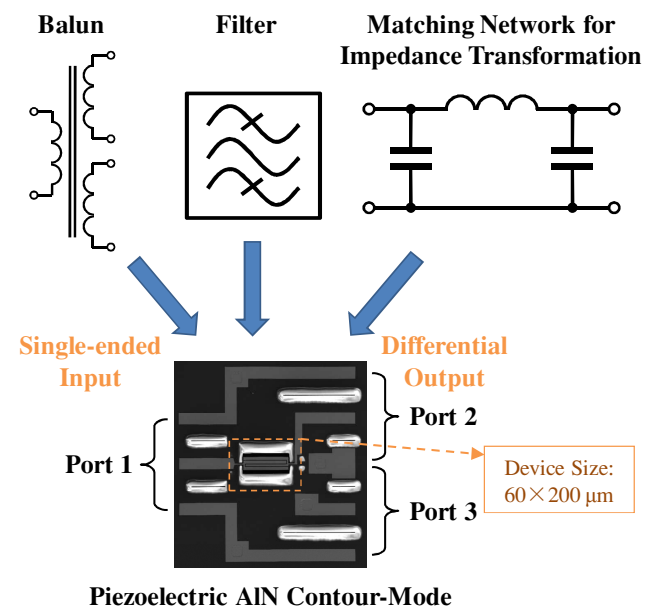

Single-ended-to-Differential Filter

Fig. 4. Schematic illustration showing how the balun, narrow-band filter, and impedance transformer functions are combined in a singleended-to-differential (S2D) AlN contour-mode MEMS filter.

Thanks to this unique feature provided by the piezoelectric AIN contour-mode RF MEMS technology (Fig. 4), the S2D AlN filters will serve as an effective bridge between the antenna $(\sim 50 \Omega)$ and the transceiver differential integrated circuits (IC, with high impedance up to $\sim \mathrm{k} \Omega$ ). The singleended port is designed to provide $50 \Omega$ matching for the antenna, while the impedance transformation ratio (which is set by the ratio of the number of sub-resonators in the different ports) can be used to define the output impedance and realize noise matching for low-noise amplifiers (LNA). The same principle also applies to power amplifier (PA) design in the transmission chain.

Although the same function can also be realized by SAW devices, they cannot be directly integrated with CMOS circuits. Traditional RF design requires a standard interface impedance of $50 \Omega$ for conjugate impedance matching to maximize power transfer and to facilitate the independent design of different components. Consequently, SAW devices usually have to be designed with $50 \Omega$ termination for standard interfacing with other components. However, for the piezoelectric AlN contourmode MEMS devices, which are also post-CMOS compatible, single-chip integrated RF solutions can be envisioned. In this case, the on-chip connections between different blocks are typically much less than the RF signal wavelength and transmission line effects can be neglected. Therefore some of the dilemmas (e.g., simultaneous impedance matching and noise optimization for LNA) encountered in conventional RF or microwave design with discrete electronic components can be addressed by abandoning the $50-\Omega$ requirement and using the integrated AlN MEMS devices. This MEMS-IC integration and co-design has the possibility to revolutionize the field of communication circuits by enabling lower power, smaller form factor, integrated, and batch-fabricated (therefore lower cost) radios implemented in unconventional RF architectures.

\section{CONLCUSION}

Multiple-frequency single-ended-to-differential (S2D) and differential-to-differential (D2D) channel-select RF MEMS filters have been demonstrated on the same chip with high performance (low insertion loss, high off-band rejection and high common-mode suppression) based on single-layer (SL) and dual-layer-stacked (DLS) AIN contour-mode resonators. The proposed S2D configuration combines the functions of a balun (single-ended to differential transformation), a narrowband filter (channel selection) and an impedance transformer in an ultra-compact MEMS structure. The demonstrated devices and proposed ideas have the potential to revolutionize the field of RF and microwave wireless communications.

\section{REFERENCES}

[1] C. T.-C. Nguyen, "MEMS Technology for Timing and Frequency Control," TUFFC, vol. 54, no. 2, pp. 251-270, 2007.

[2] N. M. Pletcher, et al., "A $2 \mathrm{GHz} 52 \mu \mathrm{W}$ Wake-Up Receiver with $-72 \mathrm{dBm}$ Sensitivity Using Uncertain-IF Architecture," ISSCC 2008, pp. 524-525, 2008.

[3] G. Piazza, et al., "One and Two Port Piezoelectric Higher Order ContourMode MEMS Resonators for Mechanical Signal Processing," Solid-State Electr., vol. 51, pp. 1596-1608, 2007.

[4] R. Ruby, et al., "PCS $1900 \mathrm{MHz}$ Duplexer using thin Film Bulk Acoustic Resonators (FBARs)," Electronics Letters, vol. 35, no. 10, pp. 794 -795, 1999.

[5] T. Jamneala, et al., "Coupled Resonator Filter with Single-Layer Acoustic Coupler," TUFFC, vol. 55, no. 10, pp. 2320-2326.

[6] S.-S. Li, et al., "An MSI Micromechanical Differential Disk-Array Filter," Transducers 2007, Lyon, France, 2007.

[7] D. Weinstein, et al., "Dielectrically Transduced Single-Ended to Differential MEMS Filter," ISSCC 2006, San Francisco, CA.

[8] G. Piazza, et al., "Piezoelectric Aluminum Nitride Vibrating ContourMode MEMS Resonators," JMEMS, vol. 15, no. 6, pp. 1406-1418, 2006.

[9] K. E. Wojciechowski, et al., "Single-Chip Precision Oscillators based on Multi-Frequency, High-Q Aluminum Nitirde MEMS Resonators," Transducers 2009, Denver, CO, USA, 2009.

[10] C. Zuo, et al., "Channel-Select RF MEMS Filters Based On Self-Coupled AIN Contour-Mode Piezoelectric Resonators," IUS 2007, New York, NY, USA, 2007.

[11] N. Sinha, et al., "Integration of AlN Micromechanical Contour-Mode Technology Filters with Three-Finger Dual Beam AIN MEMS Switches," EFTF-IFCS 2009, Besançon, France, 2009.

[12] S. Kiaei, et al., "Low-Power High-Q NEMS Receiver Architecture," ISCAS 2005, Kobe, Japan, 2005. 